\title{
Face recognition with illumination, pose and expression variations using SIFT method
}

\author{
Wei Ge ${ }^{a}$, Lijuan Cai ${ }^{b}$ and Weida Zhan ${ }^{c}$ \\ School of Electronics and Information Engineering Changchun University of Science and \\ Technology, Changchun 130000, China \\ ageweiciomp@163.com, ${ }^{\text {b }}$ cailijuan@cust.edu.cn, ${ }^{\mathrm{c}}$ zhanweida@ cust.edu.cn
}

Keywords: PIE conditions, face recognition, SIFT feature, match.

\begin{abstract}
Prior research has shown that under the illumination controlled and user cooperative conditions, nearly all of face recognition method perform very well, but when it comes to the variant illumination, pose and expression (PIE) conditions, the performance of these methods degrade seriously. Face recognition in variant PIE conditions is one of the most challenging problem in this field. In this paper, a method based on SIFT features is used to research on face recognition in variable PIE conditions. Three professional PIE-variable face database are used to experiment the performance of SIFT method. The experiment results show the huge potential of SIFT method in application to face recognition in variable PIE conditions.
\end{abstract}

\section{Introduction}

During the past several decades, face recognition has been shown great potential applications in public security, law enforcement and surveillance, digital entertainment, access control and others. Considerable progress has been made in thid field [1]. Now in controlled conditions, face recognition systems perform very well, but when it comes to the variant illumination, pose and expression (PIE) conditions, the performance of systems need to be improved.Some pointed methods are attempted to investigate the problem of face recognition in variable illumination,pose and expression conditions respectively.[2] has shown the attempt to reduce the influence of light source or a large amount of training data or some 3D face models whose facial shapes and albedos are obtained in advance, which is not practical for most real word scenarios. The 3D model methods based on illumination modeling are investigate to reduce the influence of illumination factor[3].A same problem of these method is that the computation is not efficient enough. When it comes to the adverse factors of pose and expression, an author had proposed an approach that couples the geometric information of face with the texture one[4]. In paper [5], a novel perception inspired non-metric partial similarity measure was introduced, which could help capturing the prominent partial simlilarities that were dominant in human perception. The authors proposed two methods to automatically set the similarity threshold, which were based on the general golden section rule and the maximum margin criterion.

In the actual application of face recognition systems, these adverse factors are almost concurrent, so an effective method which is applicable to the PIE variant conditions is needed to be proposed. In this paper, the Scale Invariant Feature Transform (SIFT) is proposed to dealing with above problem. SIFT features are extracted from images to help in reliable match between different views of the same object by SIFT. These features are invariant to scale and orientation, and highly distinctive of the image [6].Paper [7, 8] and [9] have shown the application of SIFT for recognizing faces in controlled situations, and performed well. This paper proved that SIFT could also perform well under variable PIE situation by some experiments based on several professional PIE-variable face database.

The rest of this paper is organized as follows. SIFT method is reviewed. Next, the application of SIFT are described, to extract SIFT features of PIE-variant faces images. Then, extensive experiments are conducted and results are analyzed. Finally, Analysis and conclusions are presented. 


\section{The Scale-invariant Feature Transform}

The scale invariant feature transform, called SIFT descriptor, has been proposed by Lowe [6], and proved to be invariant to image rotation, projective transform, scaling translation, and partly illumination changes. So the features extracted by SIFT have been shown to be invariant to image rotation and scale and robust across a substantial range of affine distortion, addition of noise, and change in illumination. The basic idea of the SIFT descriptor is detecting feature points efficiently through a staged filtering approach that indentifies stable points in the scale space. The approach is efficient on feature extraction and has the ability to identify large numbers of features.

The SIFT features are extracted in four steps [6]. The first step is to construct a Gaussian scale space function from the input image, to compute the locations of potential interest points in the image by detecting the maxima and minima of a set of Difference of Gaussian (DoG) filters applied at different scales all over the image. Secondly, these locations are localized to sub-pixel accuracy and eliminated if found to be unstable. The third step is to assign an orientation to each keypoint based on local image features. A histogram of orientations is formed from the gradient orientation at all sample points within a circular window of a feature point. Peaks in this histogram correspond to the dominant directions of each feature point [8]. These orientations, scale and location for each keypoint enable SIFT to construct a canonical view for the keypoint that is invariant to similarity transforms [10]. Finally, a local feature descriptor is computed at each key point. This descriptor is based on the local image gradient. Generally, the gradient magnitude and the orientation are smoothed by applying a Gaussian filter and then sampled over a $4 \times 4$ grid with 8 orientation planes. Then, each feature is a vector of dimension 128 distinctively identifying the neighborhood around the key point [7].

When it comes to matching feature vectors of two images, a simple Euclidean distance measure is used. A feature is considered matched with another feature when the distance to that feature is less than a specific threshold $\mathrm{T}$ of the distance to the next nearest feature. Then, the number of false matches can be reduced.

\section{SIFT Features of PIE-variant faces images}

For validating the performance of SIFT descriptors, Three professional PIE-variable face database are used [11, 12, 13, 14]. Markus Weber face database is an illumination-variable database, it contains 450 images collected in different illumination background, and offered by 27 persons [11,12].JAFFE database is an expression-variable database, which contains 213 images of 7 facial expressions ( 6 basic facial expressions +1 neutral) posed by 10 Japanese female models. Each image has been rated on 6 emotion adjectives by 60 Japanese subjects [13]. Georgia Tech face database is a PIE-variable database, which contains images of 50 people taken in two or three sessions between 06/01/99 and 11/15/99. All people in the database are represented by 15 color JPEG images with cluttered background taken at resolution $640 \times 480$ pixels. The pictures show frontal and/or tilted faces with different facial expressions, lighting conditions and scale [14].

Figure 1 illustrates that a large number of SIFT features could be extracted from face images, and most of these features are assembled on eyes, noses, and mouths. So it could be used representing the local features of faces very well. The length of red arrows represent the scale of key points, the green circles where the starting points of arrows are the location of key points, the direction of arrows represent the gradient orientation of key point corresponding to each scale.

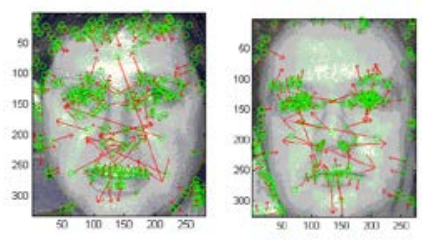

Fig. 1 SIFT features of face images 

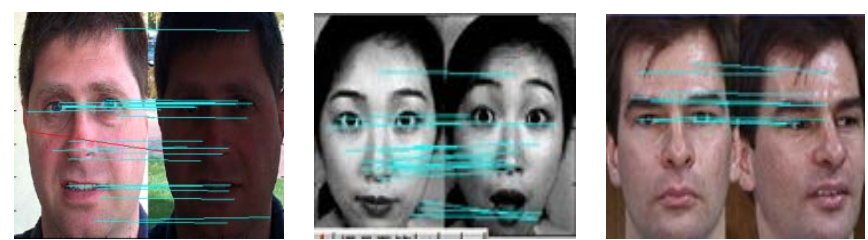

Fig. 2 Matching results for different PIE database with threshold $\mathrm{T}=0.6$

Some matching results of variable-PIE SIFT features are shown in Figure 2.The blue lines show the right matching points, while the red lines show the wrong matching points. Specially, Images of different faces are similar on the whole, they all have similar facial features, and the same expression maybe. So the SIFT vectors of two different persons may be matchable. In paper [15], we have proved that SIFT could overcome the whole comparability of different faces and extract the local detail feature of face exactly.

\section{Recognition Experiments and Results}

Three recognition experiments are conducted base on PIE databases respectively. One image per person is chosen to be sample image, other images are used to test. For example, Markus Weber database contains 450 images collected in different illumination background, and offered by 27 persons. 27 images are chosen to be sample images, other423 images are used to test. First, the SIFT features are extracted from all sample images. Then, a test face image is given, the features extracted from this image are compared against the features from each sample image. The sample image with the largest number of matching points is considered the nearest face image, and is used for the classification of the test image. Table 1 to Table 3 show the False Accept Rate (FAR) with different threshold $\mathrm{T}$ based on different face databases, and the results show that the FAR is smaller by decreasing threshold T. Specially, we haven't obtained a better result in Table 3 for the complexity of Georgia Tech face database, which contains images with different lighting, different expression and different pose conditions synthetically. For validating the availability of SIFT method in variant- PIE condition, another experiment with PCA method is conducted to contrast. First, all images in Georgia Tech face database need to be pre-processed to be the cropped images of dimensions 60X60 pixels by zooming in and zooming out. Train sets are 2 images per person, 3 images per person and 5 images per person respectively. 500 images are used to test. Table 4 shows that the FAR is more adverse when other method is used to recognize faces based on Georgia Tech face database.

Table 1 Recognition results of Markus Weber database with variant-illumination

\begin{tabular}{cccccc}
\hline $\mathrm{T}$ & $\mathrm{T}=0.60$ & $\mathrm{~T}=0.55$ & $\mathrm{~T}=0.49$ & $\mathrm{~T}=0.45$ & $\mathrm{~T}=0.40$ \\
\hline FAR(\%) & 8.27 & 6.86 & 4.96 & 4.73 & 4.73 \\
\hline
\end{tabular}

Table 2 Recognition results of JAFFE database with variant-expression

\begin{tabular}{cccccc}
\hline $\mathrm{T}$ & $\mathrm{T}=0.49$ & $\mathrm{~T}=0.45$ & $\mathrm{~T}=0.40$ & $\mathrm{~T}=0.38$ & $\mathrm{~T}=0.35$ \\
\hline FAR (\%) & 8.82 & 6.86 & 5.82 & 4.41 & 4.41 \\
\hline
\end{tabular}

Table 3 Recognition results of Georgia Tech face database with variant-PIE

\begin{tabular}{ccccc}
\hline $\mathrm{T}$ & $\mathrm{T}=0.49$ & $\mathrm{~T}=0.45$ & $\mathrm{~T}=0.40$ & $\mathrm{~T}=0.40$ \\
\hline $\mathrm{FAR}(\%)$ & 27.84 & 24.59 & 23.78 & 23.78 \\
\hline
\end{tabular}

Table 4 Recognition results of Georgia Tech database using PCA method

\begin{tabular}{cccc}
\hline Train numbers & 100 & 150 & 250 \\
\hline FAR(\%) & 42 & 37.8 & 33.6 \\
\hline
\end{tabular}




\section{Conclusion}

This paper proposes SIFT method for face recognition in variable PIE condition. The advantage of making use of SIFT algorithm is as follows: it can match and recognize face images of differ-size without normalizing face image complicatedly, and the method needn't training process, computing and experimention are all simple. Several experiments are conducted, and the results show that SIFT could overcome the difference of faces coursed by variable PIE condition and extract more useful features of face images. The recognition experimental results, performed on three professional PIE-variant face database, demonstrate the huge potential of SIFT algorithm in application to face recognition with PIE variations.

\section{References}

[1] V.Vijayakumari,Face recognition techniques: a survey, World Journal of Computer Application and Technology 1(2),(2013)41-50.

[2]Yi Zhang,Ying Chu, Xinggang Mou and Guilin Zhang, Face recognition under variable lighting using local qualitative representations, MIPPR 2007.Proc.of SPIE 6788,67881L-1L7(2007)

[3]V. Blanz, T. Vetter, Face Recognition Based on Fitting a 3D Morphable Model, IEEE Trans. Pattern Analysis and Machine Intelligence, 25(9), (2003)1-12.

[4]A.M.Mart' inez, Matching Expression Variant Faces, Vision Research 43(9) , (2003) 1047-1060.

[5]X.Y. Tan, S.C. Chen, Face Recognition Under Occlusions and Variant Expressions With Partial Similarity, IEEE Transactions on information forensics and security 4 (2) , ( 2009) 217-230.

[6]David G, Lowe, Distinctive image features from scale-invariant keypoints, International journal of computer vision60, (2004)

[7]C. Geng and X. Jiang, Face recognition using sift features, ICIP 2009, (2009)3313-3316.

[8]Dakshina Ranjan Kisku, Ajita Rattani, Face identification by SIFT-based Complete Graph Topology, 2007 IEEE Workshop on In Automatic Identification Advanced echnologies, (2007)63-68.

[9]Information on http://www.cs.st-andrews.ac.uk/ yumeng/yumeng-SIFT

[10] Information on http://www.cs.cmu.edu/ ־yke/pcasift/

[11]Weber, Markus, Face database collection of Markus Weber FaceDatabase Collection of Markus Weber, [Online] 02 February (2006).

[12] Information on http://www.vision.caltech.edu/Image_Datasets/faces/

[13]Michael J. Lyons, Miyuki Kamach , Jiro Gyoba, Japanese Female Facial Expressions (JAFFE), Database of digital images (1997).

[14] Information on http://www.anefian.com/research/face_reco.htm

[15]Wei Ge,Zhiwen Xu,Chunlei Shi, Weida Zhan, Recognition of Expression-variant Faces using SIFT Method, CICN2012, (2012)398-401 